\title{
RENDEZVOUS MANEUVERS USING GENETIC ALGORITHM
}

\author{
Denílson Paulo Souza dos Santos ${ }^{1}$, Antônio F. Bertachini de ALmeida Prado ${ }^{1}$, \\ ANDERSON RODRIGO BARRETTO TEODORO ${ }^{2}$ \\ ${ }^{1}$ INPE - National Institute for Space Research, Mechanics and Control Division, \\ Avenida dos Astronautas, 1758, São José dos Campos. \\ E-mails: denilson.paulo@gmail.com; prado@dem.inpe.br; \\ 2 ETEP / CETEC - Science and Technology Center, Computer Engineering, \\ Av. Barão do Rio Branco, 882, São José dos Campos. \\ E-mails: andersonrbteodorolgmail.com
}

\begin{abstract}
The goal of the present paper is to study orbital coplanar maneuvers between circular orbits. The control available to perform this maneuver is the application of several impulses. The solutions has searched that minimize the total variation of velocity that has to be used to implement the impulses calculated by the algorithms. The transfer time has obtained, but it isn't considered as an optimization parameter. A genetic algorithm is used to solve the problem, combined with the Lambert's Problem associated with those transfers. With the goal of making comparisons, a numerical algorithm has developed to solve the same transfers, but considering a low thrust maneuver. A propulsion system provides large savings in consumption, but with an expense of longer maneuvers.
\end{abstract}

Keywords— Astrodynamics, Rendezvous, Genetics Algorithm

\section{Introduction}

The Genetic algorithms (GA) are heuristic methods that search for optimum solutions. It has applications in several areas in order to optimize. Functions, within a specific space domain, are determined by applying methods based on the Darwinian evolution theory. In this situation, a set of possible solutions of the problem considered here may be considered as a population. Then, applying methods of crossover and mutation among individuals, the population evolves and tends to produce better individuals that represent better the solutions of the problem. The key point in this approach is to specify a measurement that can define the best individuals. Several missions can benefit from the optimization algorithm used in this work.

The main ones are: transfer with free time (to change the orbit of the space vehicle without restrictions to the time required for the execution of the maneuver); "Rendezvous" (when one desires that the space vehicle reaches and remains on the side of another space vehicle); "Flyby" (when it is desired to intercept another celestial body, however without the objective of re-maining next to it); "Swing-By" (when a close approach with a celestial body is used to gain or lose energy, velocity and angular momentum).

Studying optimal space maneuvers that searches the minimum fuel consumption for interplanetary missions is an important field of research for development of space technologies. The problem of transferring a spacecraft between two coplanar circular orbits with free time in a central force field was studied, obtaining as a solution a four-impulsive elliptical transfer orbit using to genetic algorithm (Santos et al, 2012a and $2012 b$ ). In this particular case transfer of a spacecraft from one body back to the same body (Santos et al, $2012 b$ ). The literature is extensive with respect to the problems involving transfer orbits and optimal spacecraft maneuvers (Prussing, 1979; Santos et al, 2011, Santos et al, 2012a and 2012b; Santos et. al, 2013).

\section{The genetics algorithm}

The genetic algorithm is a stochastic global search method inspired on the natural genetic and biological evolution. The genetic algorithm operates on a population of potential solutions by applying the principle of survival of the fitness to produce better and better approximations to find the best solution.

At each generation, is created new individuals by the process of selecting individuals according to their level of fitness in the problem domain and breeding them together using operators inspired in natural genetics. So, the idea is to create individuals that are better suited to their environment than the individuals that were on the previous generation.

In this case, a generation is one iteration with a certain quantity of evaluation of the objective function. In each evaluation, is applied the genetic operators, inspired in natural genetics. For the representation, the individuals are created as a chromosome where each one is considered a possible solution for the problem.

Genetic algorithms differ from traditional techniques by using probabilistic rules, not deterministic and by working with population of individuals, different of other methods that work from a single point. This is a great advantage, because by maintaining a population with good individuals, the probability of reaching a false peak is reduced.

This algorithm introduces many new concepts, including the fitness of a chromosome that represents the quality of the individual at the environment, the selection probability of an individual, the crossover operator, the mutation and the epidemic operators to introduce random perturbations in the search. In the 
Figure 1 is demonstrated an analogy to help the understanding the inspiration between genetic and computer languages.

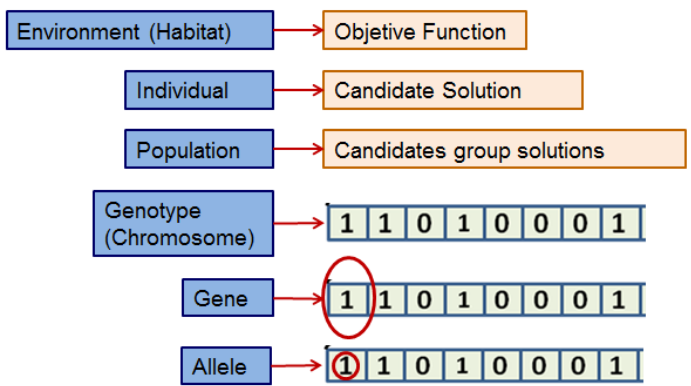

Figure 1. Demonstration of a crossing-over operator between parents.

\subsection{Crossing-Over}

The crossing-over operator is used to create new individuals from parents. This new individual is created from a random cut-off dividing the parents in two parts, having the same or different size. The first son consists on the left part of the father with the right part of the mom and the second son consists on the right part of the father with the left part of the mom. In the Figure 2 is demonstrated this schema.

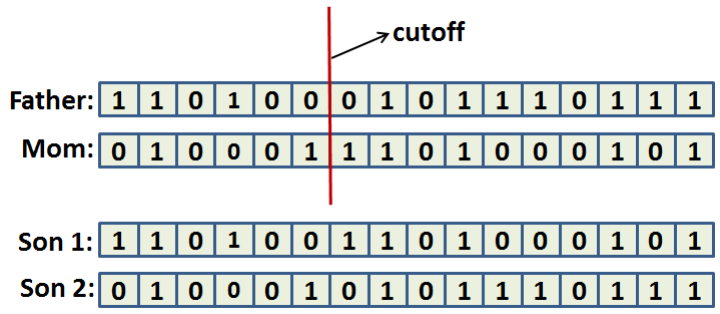

Figure 2. Demonstration of a crossing-over operator between parents.

\subsection{Mutation}

The mutation operator is used to introduce random perturbations in the search, having a low occurrence probability, around $0.5 \%$. This perturbations is created by generating a random number between 0 and 1 , if this number is smaller than the occurrence probability, the mutation operator works, flipping randomly a bit in the chromosome, if not, the mutation operator is ignored.

\subsection{Epidemic}

The epidemic is another operator used to introduce random perturbations in the search, having a very low occurrence probability, around $0.01 \%$. This perturbations is created by generating a random number between 0 and 1 , if this number is smaller than the occurrence probability, the epidemic operator works, killing a random part of the population and creating new individuals to occupy the environment (Figure 3).

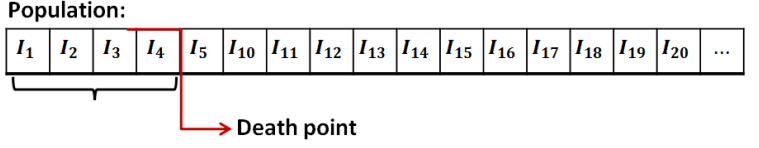

Figure 3. Epidemic killing the population.

\subsection{Function Objective}

Comparisons are made fitness of solutions to decide which should be propagated to the next generation. Normally fitness is directly related to the solution objective value, with the better objective value indicating the higher fitness. When the Genetics Algorithm (GA) procedure calls your function objective, it passes an array in the first parameter that specifies the selected solution, which is referred to as the selection parameter. The selection parameter must not be altered in any way by your function objective.

The fitness of each individual should be computed using the five data that define the problem $\left(a_{1}, e_{1}, a_{2}, e_{2}, \Delta \omega\right.$, the first being unit because of the normalization) and the three genes $\left(v_{1}, \Delta v, y\right)$ that characterize the individual. One obtains, in sequence: the true anomaly of the arrival point

$$
v_{i}=v_{i-1}+\Delta v
$$

the radii of the departure and arrival point are given by

$$
\begin{aligned}
& r_{1}=\frac{a_{1}\left(1-e_{1}^{2}\right)}{1+e_{1} \cos v_{1}} \\
& r_{2}=\frac{a_{2}\left(1-e_{2}^{2}\right)}{1+e_{2} \cos \nu_{2}}
\end{aligned}
$$

the distance between $\mathrm{P}_{1}$ and $\mathrm{P}_{2}$, on each arc, is

$$
c=\sqrt{r_{1}^{2}+r_{2}^{2}-2 r_{1} r_{2} \cos \Delta v}
$$

the semi-major axis of the transfer orbit is

$$
a_{\min }=\frac{r_{1}+r_{2}+c}{4}
$$

the distances $\mathrm{c}_{1}$ and $\mathrm{c}_{2}$ of $\mathrm{P}_{1}$ and $\mathrm{P}_{2}$ from the vacant focus $\mathrm{F}_{2}$ can be specified by the equations

$$
c_{i}=2 a-r_{i}
$$

The Figure 4 shows a description of several important variables.

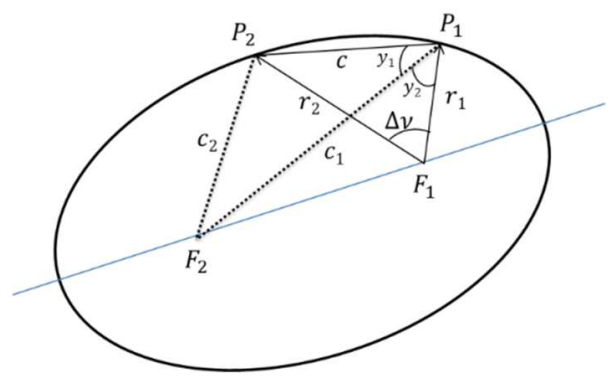

Figure 4 - Geometry of the Problem and the angles involved in the problem. 
The angles is calculated by

$$
\begin{gathered}
y=\operatorname{arcos}\left(\frac{r_{1}^{2}-r_{2}^{2}+c^{2}}{2 r_{1} c}\right) \\
y_{1}=\operatorname{arcos}\left(\frac{c_{1}^{2}-c_{2}^{2}+c^{2}}{2 c_{1} c}\right)
\end{gathered}
$$

the eccentricity of the transfer orbit is given by

$$
e_{t}=\frac{\sqrt{c_{1}^{2}+r_{1}^{2}-2 c_{1} r_{1} \cos \gamma_{2}}}{2 a_{t}}
$$

the true anomaly $\theta_{1}$ of the $\mathrm{P}_{1}$ on the transfer orbit is

$$
\theta_{1}=\operatorname{arcos}\left(\frac{a_{t}\left(1-e_{t}^{2}\right)-r_{1}}{r_{1} e_{t}}\right)
$$

the argument of perigee for the transfer orbit is

$$
\omega=v_{1}-\theta_{1}
$$

which is the angle between the perigees of the transfer and the initial orbits.

The geometry of the maneuvers has been measured. One calculates the radial and the tangential components of the spacecraft velocity before and after both impulses, what permits the computation of the total $\Delta V$, which has been assumed as the measure of the individual fitness. Non-dimensional variables are used in the code (Santos et al, 2012a and 2012b) They are shown below.

$$
\begin{gathered}
r=\frac{\tilde{r}}{\tilde{a}_{1}} \\
v=\frac{\tilde{v}}{\sqrt{\frac{\mu}{\tilde{a}_{1}}}}
\end{gathered}
$$

The distances and velocities (reference) are the semimajor axis of the initial orbit and the velocity on a circular orbit with the same energy as the initial one. The reference time is therefore $\sqrt{\frac{\tilde{a}_{1}^{3}}{\mu}}$.

\section{Numerical solutions}

Genetic algorithms are iterative schemes where, in each iteration, the population is modified, using the best features of the elements of the previous generations. They are subjected to five basic operations to produce better results:

To apply the genetic algorithms in orbital maneuvers, an impulsive hypothesis is used for the engine of the spacecraft. It means that the velocity is assumed to change in zero time and a sequence of keplerian orbits represents the motion of the spacecraft. More details about this type of maneuver can be seen (Santos et al, 2012a and 2012b)

Then, the optimization method using genetic algorithms was used and several missions were simulated. Some of them are shown in more details, with initial radii $r_{o}=1$ and final radius $r_{f}=2$ and $r_{f}=3$. The results are shown in Table 1 and Figures 5 to 8.

Table 1 - Maneuvers between coplanar circular orbits showing the values of the $\Delta \mathrm{V}$.

\begin{tabular}{|c|c|c|c|c|}
\hline $\mathbf{N o}$ & $\mathbf{1}$ & $\mathbf{2}$ & $\mathbf{3}$ & $\mathbf{4}$ \\
\hline $\begin{array}{c}\text { Simulation } \\
(\mathbf{r o = 1})\end{array}$ & $\mathbf{r}_{\mathbf{f}}=\mathbf{2}$ & $\mathbf{r}_{\mathbf{f}}=\mathbf{3}$ & $\mathbf{r}_{\mathbf{f}}=\mathbf{1 . 2}$ & $\mathbf{r}_{\mathbf{f}}=\mathbf{1 . 5}$ \\
\hline $\boldsymbol{\Delta} \mathbf{V}_{\mathbf{1}}$ & 0.023829 & 0.000016 & 0.035322 & 0.007858 \\
\hline $\boldsymbol{\Delta} \mathbf{V}_{\mathbf{2}}$ & 0.144021 & 0.224097 & 0.017854 & 0.000000 \\
\hline $\boldsymbol{\Delta} \mathbf{V}_{\mathbf{3}}$ & 0.011422 & 0.002581 & 0.068397 & 0.088876 \\
\hline $\boldsymbol{\Delta} \mathbf{V}_{\mathbf{4}}$ & 0.108731 & 0.178097 & 0.074701 & 0.084990 \\
\hline $\boldsymbol{\Delta} \mathbf{V}_{\mathbf{T}}$ & 0.288004 & 0.404792 & 0.196275 & 0.181724 \\
\hline $\begin{array}{c}\text { Hohmann } \\
\boldsymbol{\Delta} \mathbf{V}\end{array}$ & 0.284457 & 0.393847 & 0.086947 & 0.181645 \\
\hline
\end{tabular}

In particular, optimal rendezvous between two coplanar orbits, with maneuvers using up to 4 burns, were used to generate those results. Rendezvous is a maneuver where a space vehicle needs to encounter a second one that is in a different orbit. Some more information regarding rendezvous maneuver can be seen in Santos and Prado (2012b). Figure 3 shows the states of the genetic algorithm and its evolution after 500 evaluations of the objective function. The genetic algorithm provided satisfactory solutions when compared with the solutions of the literature. The population comprises of 500 individuals and up to 250 generations of individuals in those simulations. Maneuver 2 shows an interesting feature of the algorithm. Although the specified number of impulses was four, the algorithm found a better solution that uses only three impulses, so it generated an impulse with zero cost.

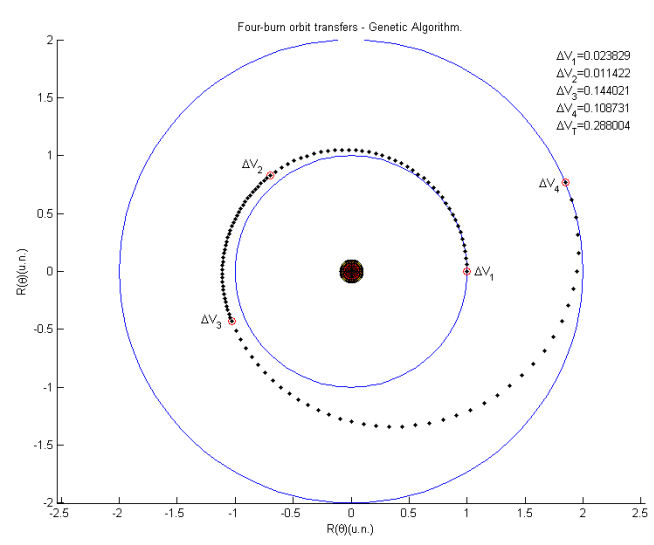

Figure 5. Four-burn orbital rendezvous, simulation 1:

$$
\mathrm{r}_{0}=1, \mathrm{r}_{\mathrm{f}}=2
$$




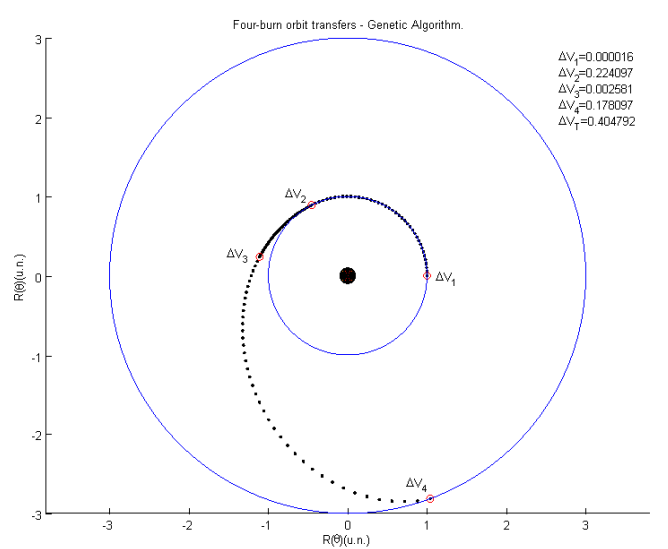

Figure 6. Four-burn orbital rendezvous, simulation 2: $\mathrm{r}_{0}=1, \mathrm{r}_{\mathrm{f}}=3$

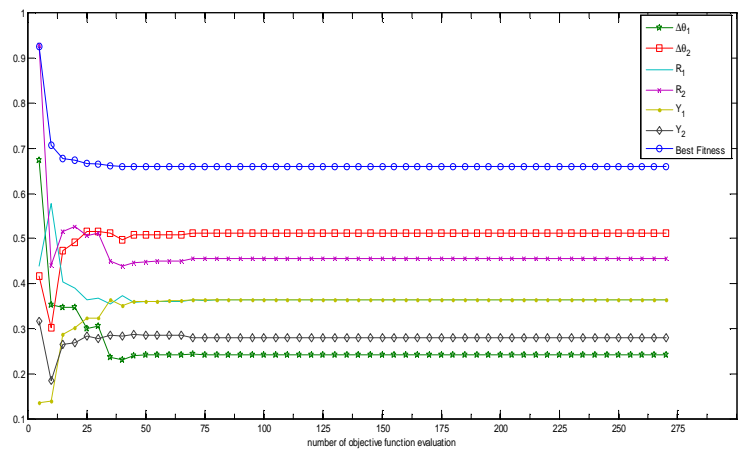

Figure 7 - The variables of the problem and the best fitness, simulation 1 , with 4 burns.

After that, a different situation was assumed to test the Genetic Algorithm. Intermediate constraints were added to force the spacecraft to pass by several intermediate orbits before reaching the final desired one. To solve the problem, the engine was assumed to be also impulsive, but the number of impulses was increased to allow the satisfaction of the intermediate constraints (passing by specific orbits) (Table 2, Figure 7 and 8).

Table 2 - Septa-burn orbit transfers

\begin{tabular}{|l|c|c|c|c|}
\cline { 3 - 5 } \multicolumn{1}{c|}{} & \multirow{2}{*}{$\begin{array}{c}\mathbf{r}_{\mathbf{0}}=\mathbf{1}, \mathbf{e}=\mathbf{0} \\
\mathbf{r}=\mathbf{3}\end{array}$} & \multicolumn{3}{|c|}{ Keplerian transfer orbits } \\
\cline { 3 - 5 } \multicolumn{1}{c|}{$\boldsymbol{\Delta} \mathbf{V}_{\mathbf{1}}$} & 0.044278 & 1.099522 & 0.090514 & 6.278946 \\
\hline $\boldsymbol{\Delta} \mathbf{V}_{\mathbf{2}}$ & 0.000726 & 1.101127 & 0.089776 & 0.010485 \\
\hline $\boldsymbol{\Delta} \mathbf{V}_{\mathbf{3}}$ & 0.149698 & 1.599995 & 0.250019 & 3.132906 \\
\hline $\boldsymbol{\Delta} \mathbf{V}_{\mathbf{4}}$ & 0.512103 & 1.854493 & 0.474787 & 2.206169 \\
\hline $\boldsymbol{\Delta} \mathbf{V}_{\mathbf{5}}$ & 0.000000 & 1.854493 & 0.474787 & 2.206169 \\
\hline $\boldsymbol{\Delta} \mathbf{V}_{\mathbf{6}}$ & 0.026218 & 2.091447 & 0.530214 & 2.270242 \\
\hline $\boldsymbol{\Delta} \mathbf{V}_{\mathbf{7}}$ & 0.223406 & \multicolumn{3}{|c|}{ final circular orbit } \\
\hline $\boldsymbol{\Delta} \mathbf{V}_{\mathbf{T}}$ & 0.956429 & \multicolumn{4}{|c|}{} \\
\hline
\end{tabular}

These solutions differ from each other by intermediate constraints that we used, so the solutions have different transfer times and the trajectories of the spacecraft passes by different regions of the space, to be able to accomplish different goals for the mission.

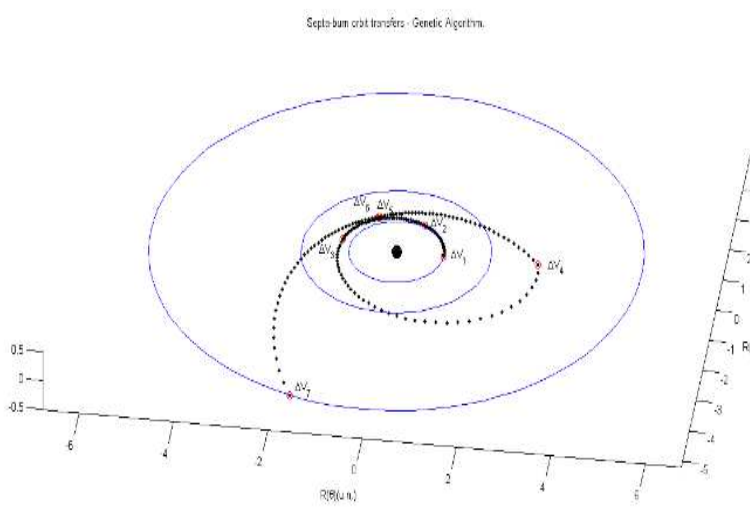

Figure 8. Seven-burn orbit transfers. $r_{0}=1$ (Earth). $r_{f}$ $=5.202803$ (Jupiter). Solution 1. with 7 burns and $\Delta \mathrm{V}_{\mathrm{T}}=1.407184$

Then we implemented a low thrust algorithm. This type of maneuver assumes that the engine can deliver a continuous but low thrust to the spacecraft. Similar problems are studied in several papers in the literature, like shown in Santos and Prado (2012b). The main advantage is that it consumes a lot less fuel, but at an expense of larger times for the maneuver as well as more complex implementation of the hardware. The choice of which technique to use depends on the mission requirements and on the engines available for the spacecraft.

In the present formulation, the main ideas shown in Biggs [apud Santos, 2012b] are used and to avoid singularities in the equations of motion, the following variables are used:

$$
\begin{gathered}
X_{1}=\sqrt{\frac{a\left(1-e^{2}\right)}{\mu}} \\
X_{2}=\operatorname{ecos}(\omega-\phi) \\
X_{3}=\operatorname{esin}(\omega-\phi) \\
X_{4}=(\text { Fuel Consumed }) / m_{0} \\
X_{5}=t \\
X_{6}=\cos \left(\frac{i}{2}\right) \cos \left(\frac{\Omega+\phi}{2}\right) \\
X_{7}=\sin \left(\frac{i}{2}\right) \cos \left(\frac{\Omega-\phi}{2}\right) \\
X_{8}=\sin \left(\frac{i}{2}\right) \sin \left(\frac{\Omega-\phi}{2}\right) \\
X_{9}=\sin \left(\frac{i}{2}\right) \sin \left(\frac{\Omega+\phi}{2}\right) \\
\phi=f+\omega-s
\end{gathered}
$$

where:

$\mathrm{a}=$ semi-major axis; $\mathrm{e}=$ eccentricity $; \mathrm{i}=$ inclination; $\Omega=$ argument of the ascending node; $\omega=$ ar- 
gument of perigee; $\mathrm{f}=$ true anomaly; $\mathrm{s}=$ range angle; $\mu$ =gravitational constant; $\mathrm{m}_{0}=$ initial mass of the spacecraft.

In those variables, the equations of motion are:

$$
\begin{aligned}
& \frac{d X_{1}}{d s}=f_{1}=S i X_{1} F_{1} \\
& \frac{d X_{2}}{d s}=f_{2}=\operatorname{Si}\{[(G a+1) \cos (s) \\
& \left.+X_{2}\right] f_{1} \\
& \left.+\mathrm{GaF}_{2} \sin (s)\right\} \\
& \frac{d X_{3}}{d s}=f_{3}=S i\{[(G a+1) \sin (s) \\
& \left.+X_{3}\right] f_{1} \\
& \left.-G a F_{2} \cos (s)\right\} \\
& \frac{d X_{4}}{d s}=f_{4}=\frac{\operatorname{SiGaF}\left(1-X_{4}\right)}{X_{1} W} \\
& \frac{d X_{5}}{d s}=f_{5}=\frac{\operatorname{SiGa}\left(1-X_{4}\right) m_{0}}{X_{1}} \\
& \frac{d X_{6}}{d s}=f_{6} \\
& =-\frac{\operatorname{SiF}_{3}\left[X_{7} \cos (s)+X_{8} \sin (s)\right]}{2} \\
& \frac{d X_{7}}{d s}=f_{7} \\
& =\frac{\operatorname{SiF}_{3}\left[X_{6} \cos (s)+X_{9} \sin (s)\right]}{2} \\
& \frac{d X_{8}}{d s}=f_{8} \\
& =\frac{\operatorname{SiF}_{3}\left[X_{9} \cos (s)+X_{6} \sin (s)\right]}{2} \\
& \frac{d X_{9}}{d s}=f_{9} \\
& =\frac{\operatorname{SiF}_{3}\left[X_{7} \sin (s)+X_{8} \cos (s)\right]}{2}
\end{aligned}
$$

Where:

$$
\begin{gathered}
G a=1+X_{2} \cos (s)+X_{3} \sin (s) \\
S i=\frac{\left(\mu X_{1}^{4}\right)}{\left[G a^{3} m_{0}\left(1-X_{4}\right)\right]}
\end{gathered}
$$

$\mathrm{F}, \mathrm{F}_{1}, \mathrm{~F}_{2}$ and $\mathrm{F}_{3}$ are the forces generated by the thrust. given by:

$$
\begin{gathered}
\vec{F}=\vec{F}_{1}+\vec{F}_{2}+\vec{F}_{3} \\
F=|\vec{F}| \\
F_{1}=F \cos (\alpha) \cos (\beta) \\
F_{2}=F \sin (\alpha) \cos (\beta) \\
F_{3}=F \sin (\beta)
\end{gathered}
$$

where $\alpha$ is the angle between the perpendicular to the radius vector and the direction of the thrust and $\beta$ is the out-of-plane angle of the thrust. The equations for the Lagrange multipliers are:

$$
\begin{aligned}
& \frac{d p_{1}}{d s} \\
& =-\frac{4 \sum_{j=1}^{9} p_{j} f_{j}+p_{1} f_{1}-p_{4} f_{4}-p_{5} f_{5}}{X_{1}} \\
& \frac{d p_{2}}{d s} \\
& =\frac{\cos (s)}{G a}\left[3 \sum_{j=1}^{9} p_{j} f_{j}-p_{4} f_{4}-p_{5} f_{5}\right] \\
& -\operatorname{Sip}_{2} F_{1}-\operatorname{Sicos}^{2}(s)\left(p_{2} F_{1}-p_{3} F_{2}\right) \\
& --\operatorname{Sicos}(s) \sin (s)\left(p_{2} F_{2}+p_{3} F_{1}\right) \\
& \frac{d p_{3}}{d s}=\frac{\sin (s)}{G a}\left[3 \sum_{j=1}^{9} p_{j} f_{j}-p_{4} f_{4}-p_{5} f_{5}\right] \\
& -\operatorname{Sip}_{3} F_{1} \\
& -\operatorname{Sicos}(s) \sin (s)\left(p_{2} F_{1}\right. \\
& \left.-p_{3} F_{2}\right) \\
& -\operatorname{Sisin}^{2}(s)\left(p_{2} F_{2}+p_{3} F_{3}\right) \\
& \frac{d p_{4}}{d s}=-\left[\frac{\sum_{j=1}^{9} p_{j} f_{j}-p_{4} f_{4}-p_{5} f_{5}}{m_{0}\left(1-X_{4}\right)}\right] \\
& \frac{d p_{5}}{d s}=0 \\
& \frac{d p_{6}}{d s}=\frac{-S i F_{3}\left[p_{7} \cos (s)+p_{8} \sin (s)\right]}{2} \\
& \frac{d p_{7}}{d s}=\frac{\operatorname{SiF}_{3}\left[p_{6} \cos (s)-p_{9} \sin (s)\right]}{2} \\
& \frac{d p_{8}}{d s}=\frac{S i F_{3}\left[p_{6} \sin (s)+p_{9} \cos (s)\right]}{2} \\
& \frac{d p_{9}}{d s}=\frac{-S i F_{3}\left[p_{8} \cos (s)-p_{7} \sin (s)\right]}{2}
\end{aligned}
$$

The control to be applied to the spacecraft can also benefit from a substitution of variables to avoid numerical problems. The following set of variables is used:

$$
\begin{gathered}
u_{1}=s_{0} \\
u_{2}=\left(s_{f}-s_{0}\right) \cos \left(\beta_{0}\right) \cos \left(\alpha_{0}\right) \\
u_{3}=\left(s_{f}-s_{0}\right) \cos \left(\beta_{0}\right) \sin \left(\alpha_{0}\right) \\
u_{4}=\left(s_{f}-s_{0}\right) \sin \left(\beta_{0}\right) \\
u_{5}=\alpha^{\prime} \\
u_{6}=\beta^{\prime}
\end{gathered}
$$

First order necessary conditions for the optimal problem can be written. For every instant of time, we have:

$$
\begin{aligned}
& \sin (\alpha)=\frac{q_{2}}{S^{\prime}} \\
& \sin (\beta)=\frac{q_{3}}{S^{\prime \prime}} \\
& \cos (\alpha)=\frac{q_{1}}{S^{\prime}}
\end{aligned}
$$




$$
\cos (\beta)=\frac{S^{\prime}}{S^{\prime \prime}}
$$

where:

$$
\begin{gathered}
S^{\prime}= \pm \sqrt{q_{1}^{2}+q_{2}^{2}} \\
S^{\prime \prime}= \pm \sqrt{q_{1}^{2}+q_{2}^{2}+q_{3}^{2}} \\
q_{1}=p_{1} X_{1}+p_{2}\left[X_{2}+(G a+\right. \\
1) \cos (s)]+p_{3}\left[X_{3}+(G a+\right. \\
1) \sin (s)] \\
q_{2}=p_{2} G a \sin (s)-p_{3} \operatorname{Gacos}(s) \\
q_{3}=-p_{6} \frac{\left[X_{7} \cos (s)+X_{8} \sin (s)\right]}{2}+ \\
p_{7}\left[\mathrm{X}_{6} \cos (\mathrm{s})-\mathrm{X}_{9} \sin (\mathrm{s})\right]+ \\
p_{8}\left[\mathrm{X}_{6} \sin (\mathrm{s})-\mathrm{X}_{9} \cos (\mathrm{s})\right]+ \\
p_{9}\left[\mathrm{X}_{7} \sin (\mathrm{s})-\mathrm{X}_{8} \cos (\mathrm{s})\right]
\end{gathered}
$$

It is also possible to include constraints. Some of the most used ones can be represented by:

$$
\begin{gathered}
S(.) \geq 0 \\
\frac{\left(a-a^{*}\right)}{\left|a-a^{*}\right|}=0 \\
\frac{\left[a(1+e)-a^{*}\left(1+e^{*}\right)\right]}{\left|a\left(1+e_{0}\right)-a^{*}\left(1+e^{*}\right)\right|}=0 \\
\frac{\left(i-i^{*}\right)}{\left|i-i^{*}\right|}=0 \\
\frac{\left(\Omega-\Omega^{*}\right)}{\left|\Omega-\Omega^{*}\right|}=0 \\
\frac{\left(\omega-\omega^{*}\right)}{\left|\omega-\omega^{*}\right|}=0
\end{gathered}
$$

\begin{tabular}{|c|c|c|}
\hline \multirow[b]{2}{*}{$n^{\circ}$} & \multirow[b]{2}{*}{$\begin{array}{c}\text { Simulation } \\
\quad\left(r_{0}=1\right)\end{array}$} & Consumption \\
\hline & & Low Thrust Maneuvers \\
\hline 1 & $\mathrm{r}_{\mathrm{f}}=2$ & 0.0886 \\
\hline 2 & $\mathrm{r}_{\mathrm{f}}=3$ & 0.1271 \\
\hline 3 & $\mathrm{r}_{\mathrm{f}}=1.2$ & 0.0831 \\
\hline 4 & $\mathrm{r}_{\mathrm{f}}=1.5$ & 0.0865 \\
\hline
\end{tabular}

The first one represents generic inequality constraints, while the other five is used to specify an orbit. After the implementation of this technique, the simulations showed in Table 1 was considered again this time using this low thrust approach. The results are shown in Table 3. The consumptions are much lower, but it is necessary to have in mind that this situation is normal and constitute the most important characteristics of low thrust engines.

Table 3 - Maneuvers between coplanar circular orbits using low thrust.

\section{Conclusion}

From the analysis of the results obtained, the genetic algorithm implemented here showed that this technique can obtain results for the proposed four impulsive rendezvous maneuvers. It means that it can be used in real cases. The algorithm is able to find solutions with a smaller number of impulses by making one or more of them with zero magnitude, if a maneuver with a lower number of impulses can be used.

It also can generate results in situations where intermediate constraints of passing by specific orbits are included. In this case, several burns are required and the consumption is larger as expected. In the examples used here the number of impulses reached the number of seven and two solutions were found by considering two different sets of intermediate constraints.

Then, a low thrust was used for the rendezvous missions. It shows the importance of this approach, which can find solutions with much lower fuel consumption, although it has some disadvantages like more time required for the maneuvers and more complex implementation of the hardware.

\section{Acknowledgments}

This work was accomplished with the support of São Paulo State Science Foundation (FAPESP) under Contracts 2009/16517-7 and INPE - Brazil.

\section{References}

Prussing. J. E.. "Geometrical Interpretation of the Angles $\alpha$ and $\beta$ in Lambert's Problem". Journal of Guidance. Control. and Dynamics. Vol. 2. No.5. 1979. pp. 442. 443.

Santos, D.P.S., Prado, A.F.B.A.P, Colasurdo, G,. "Four-Impulsive Rendezvous Maneuvers for Spacecrafts in Circular Orbits Using Genetic Algorithms". Mathematical Problems in Engineering (Print), v. 2012, p. 1-16, 2012a. DOI: 10.1155/2012/493507

Santos, D.P.S., Prado, A.F.B.A.P,. "Minimum Fuel Multi-Impulsive Orbital Maneuvers Using Genetic Algorithms". In: 1st IAA Conference on Dynamics and Control of Space Systems, 2012, Porto - Portugal. IAA-AAS-DyCoSS1-11-01, 2012b.

Santos, D.P.S., Prado, A.F.B.A.P, Colasurdo, G, Rendezvous Maneuvers with Minimal $\Delta V$. In: Recent Researches In Power Systems And Systems Science, 2011, Penang, Malaysia. Icossse'11 Proceedings Of The 10th WSEAS International Conference On System Science And Simulation In Engineering, 2011. P. 38-42.

Santos. D. P. S; Prado. A. F. B. A; Rocco., "The Study of the Asymmetric Multiple Encounters Problem and Its Application to Obtain Jupiter Gravity Assisted Maneuvers," Mathematical Problems in Engineering, vol. 2013, Article ID 745637, 12 pages, 2013. doi:10.1155/2013/745637. DOI: $10.1155 / 2013 / 745637$ 